\title{
Resonance axial-vector mass from experiments on neutrino-hydrogen and neutrino-deuterium scattering
}

\author{
Igor D. Kakorin $\circledast^{1, *}$ and Konstantin S. Kuzmin $\oplus^{1,2, \uparrow}$ \\ ${ }^{1}$ N. N. Bogoliubov Laboratory of Theoretical Physics, Joint Institute for Nuclear Research, \\ RU-141980 Dubna, Russia \\ ${ }^{2}$ A. I. Alikhanov Institute for Theoretical and Experimental Physics of NRC "Kurchatov Institute", \\ $R U-117218$ Moscow, Russia
}

(Received 23 March 2021; accepted 28 September 2021; published 2 November 2021)

\begin{abstract}
We analyze all available experimental data on the $\nu_{\mu}$ and $\bar{\nu}_{\mu}$ total and differential cross sections of charged-current single pion production through the decay of intermediate nucleon and baryon resonances measured on hydrogen and deuterium targets in the accelerator experiments at ANL, BNL, FNAL, and CERN. These data are used to determine the current "resonance" axial-vector mass of the nucleon and to fine tune the nonresonance noninterfering background contribution which described within the Rein-Sehgal approach. For this analysis, we revise the phenomenological model and the experimental dataset for the fits, modify the method of likelihood analysis compared to the previous study. The obtained model parameters coming in combination with a revised strategy for the normalization of the Breit-Wigner distributions are slightly different from the values used by default.
\end{abstract}

DOI: $10.1103 /$ PhysRevD.104.093001

\section{INTRODUCTION}

A precise calculation of the neutrino-nucleon scattering cross sections is extremely important for a correct interpretation of the results in studying neutrino properties in atmospheric and accelerator experiments [1-10]. The cross sections from charged current quasielastic scattering (CC $\mathrm{QES}), 1 \pi$-production reactions through the decay of baryon and nucleon resonances, and deep inelastic scattering are crucial for the few-GeV neutrino experiments since all the contributions are comparable in the neutrino energy range about $1 \mathrm{GeV}[11,12]$.

For the phenomenological description of the resonance $1 \pi$-production reactions, we use the phenomenological Ravndal's model [13], which was revised and modified by Rein and Sehgal in 1981 (RS model) [14,15], and other authors in subsequent years. The RS model is based on the relativistic harmonic-oscillator quark model in the formulation by Feynman, Kislinger, and Ravndal (FKR) [16] taking into account contributions from eighteen interfering low-lying nucleon and baryon resonances with masses

\footnotetext{
*Kakorin@jinr.ru

†KKuzmin@theor.jinr.ru
}

Published by the American Physical Society under the terms of the Creative Commons Attribution 4.0 International license. Further distribution of this work must maintain attribution to the author(s) and the published article's title, journal citation, and DOI. Funded by SCOAP ${ }^{3}$. $\lesssim 2 \mathrm{GeV}$ and a noninterfering nonresonance contribution as a background (NRB) for resonance reactions. The FKR approach has been adopted for the RS model in the assumption of the standard dipole parametrization for the vector and axial-vector transition form factors,

$$
G^{V, A}\left(Q^{2}\right) \propto\left(1+\frac{Q^{2}}{4 M_{N}^{2}}\right)^{1 / 2-n}\left(1+\frac{Q^{2}}{M_{V, A}^{2}}\right)^{-2},
$$

with the "standard" value of the vector mass $M_{V}=$ $0.84 \mathrm{GeV}$ and $n$-number of excitation in the resonance. In the original version of the RS model the resonance axial mass was chosen as $M_{A}=0.95 \mathrm{GeV}$, which is the average result of ANL 12-foot bubble chamber [17] and CERN Gargamelle [18,19] experiments to study QES $\nu_{\mu}$ and $\bar{\nu}_{\mu}$ reactions. In our previous study [20], the value of $M_{A}$ was obtained from the global likelihood analysis of all available experimental data known at that time from the measurement of the total cross sections of the $1 \pi$-production reactions with a variety of nuclear targets. The world average value of $M_{A}=1.12 \pm 0.03 \mathrm{GeV}$ obtained in that analysis is used in several Monte Carlo neutrino generators as a default or an option [21-25]. In the present paper, we propose a new global fit of $M_{A}$ based on the clarified phenomenological model, improved method of likelihood analysis, and the revised experimental dataset. Let us recall that the parameter $M_{A}$ is purely phenomenological and differs for different reaction types and different models of 
the electromagnetic form factors of the nucleon and the parametrization of the axial-vector form factor.

The original version of the RS model neglects the mass of the final charged lepton. This feature of the model is not a significant disadvantage for the description of the $\nu_{e}, \bar{\nu}_{e}, \nu_{\mu}$, and $\bar{\nu}_{\mu}$ reactions but is a crucial gap for the description of the $\nu_{\tau}$ and $\bar{\nu}_{\tau}$ reactions (it should be noted that Rein and Sehgal proposed the model at the end of the 1970s, and at that time the possibility of experimentally detecting the $\nu_{\tau}$ or $\bar{\nu}_{\tau}$ reaction was not discussed). In 2004, a model was adopted to calculate the cross sections of the $\nu_{\tau}$ and $\bar{\nu}_{\tau}$ by using the covariant form of the charged leptonic current with a definite lepton helicity, keeping the hadronic current unchanged (the so-called extended RS model or KLN model) [26,27]. Inclusion of the final charged lepton mass leads to a slight decreasing of the cross sections in the case of light leptons and to a notable one in the case of the $\tau$-lepton. In 2006, Berger and Sehgal took into account the pion-pole contribution to the hadronic axial current (KLN-BS model) [28]. The pion-pole contribution reduces the cross sections by a few percent in the neutrino energy range at the threshold of the reactions and decreases rapidly with increasing neutrino energy. In 2008, Graczyk and Sobczyk investigated different approaches to accounting for the nonzero mass of the charged lepton and the modification of the axial current due to the pion-pole term. It was shown that their result is equivalent to the predictions of the KLN-BS model. Alternative vector and axial form factors are proposed to improve the RS approach in the $\Delta(1232)$ resonance region [29,30]. In 2018, Kabirnezhad [31] following the original paper by Rein [15] and ideas of Hernández, Nieves, and Valverde [32], suggested a new approach for calculating the interfering NRB for the RS model [33-35]. This modification of the Rein model contains 17 interfering resonances with masses below $2 \mathrm{GeV}$ [the $F_{17}(1900)$ is excluded from the model because evidence of its existence appears only in PDG 2020 [36] and it does not have a significant effect on calculations].

Rein and Sehgal represented NRB by a resonance amplitude of $P_{11}$ character (like the nucleon) with the Breit-Wigner factor replaced by an adjustable constant $f_{\text {NRB }}$. The corresponding cross section of NRB reactions is added incoherently to the cross sections of resonance productions, assuming that NRB contributions are smooth in the phase-spacelike kinematic region and affect only the cross section of final states with $I=1 / 2$. Ignoring subtle effects of coherence NRB is regulated by $f_{\mathrm{NRB}}$ only. The problem is that the value of $f_{\mathrm{NRB}}$ cannot be predicted theoretically in the RS approach. Probably, the condition of $f_{\mathrm{NRB}}=1$ was chosen by the authors of the model to optimize the description of selected experimental data relevant at that time. Therefore, $f_{\mathrm{NRB}}$ as well as
$M_{A}$ should be defined from the modern global fits of experimental data.

The noninterfering NRB is determined by one more free phenomenological parameter $W_{\mathrm{NRB}}$, which is the bound for the invariant mass of the final hadron system, $W$, separating the kinematic region of the resonance $1 \pi$ production and deep inelastic reactions. In other words, the parameter $W_{\mathrm{NRB}}$ controls the applicability range of NRB contributions in the RS model. The parameter $W_{\mathrm{NRB}}$, as well as $f_{\mathrm{NRB}}$, is not based on the theory and is fixed in the RS model by default to a sufficiently large asymptotic value of $2.5 \mathrm{GeV}$. The reasonable value of $W_{\mathrm{NRB}}$ is important for describing experimental data measured without cuts for $W$. Furthermore, the dependence of the cross sections on the parameter $W_{\mathrm{NRB}}$ is significant for neutrino energies above $\sim 10 \mathrm{GeV}$. There are no reliable experimental data for the cross sections of the resonance $1 \pi$-production reactions measured without cut for $W$ in the neutrino energy range above a few tens of $\mathrm{GeV}$. So the parameter $W_{\mathrm{NRB}}$ cannot be reliably obtained from the global fit. For all the fits the value of $W_{\mathrm{NRB}}$ is chosen to be the default value.

Theoretical uncertainties of predicted NRB reactions and model-dependent nuclear effects in neutrinonucleus interactions complicate the physical interpretation of the phenomenological parameter $M_{A}$ obtained from the global fit of the experimental data measured on a variety of nuclear targets. To avoid this complication, we use the experimental data measured only on hydrogen and deuterium targets. In the RS approach, the $\nu_{\mu} p \rightarrow \mu^{-} \Delta^{++}, \nu_{\mu} p \rightarrow \mu^{-} p \pi^{+}$, and $\bar{\nu}_{\mu} n \rightarrow$ $\mu^{+} n \pi^{-}$reactions do not require NRB for a description of the experimental data in contrast with all other resonance $1 \pi$-production reactions. We suggest using $M_{A}$ obtained from the global fit for the cross sections of the $\nu_{\mu}$ and $\bar{\nu}_{\mu}$ reactions not requiring NRB. Using the world average value of $M_{A}$, we found the adjustable constant $f_{\mathrm{NRB}}$ from the global fit with a fixed value of $M_{A}$ for the experimental data on the cross sections of the reactions requiring NRB.

The version of the KLN-BS model implemented into the GENIE Monte Carlo neutrino event generator currently does not take into account the interference of the amplitudes of resonances with the same spin and orbitalangular momentum. Neutrino interaction with nuclei leads to the generation of hadron resonances with different quantum numbers. The amplitudes of resonances have to interfere to produce the final state of the hadron system under consideration. Each of the interfering resonances, by simultaneous decay, produces the same final system with one or several pions at a fixed invariant final mass $W$. Figure 1 illustrates the effect of interference for the total cross sections of the reactions on isoscalar nucleons. The interference effect is highly reliant on the final hadronsystem mass and is equal to a few per cent for all neutrino 


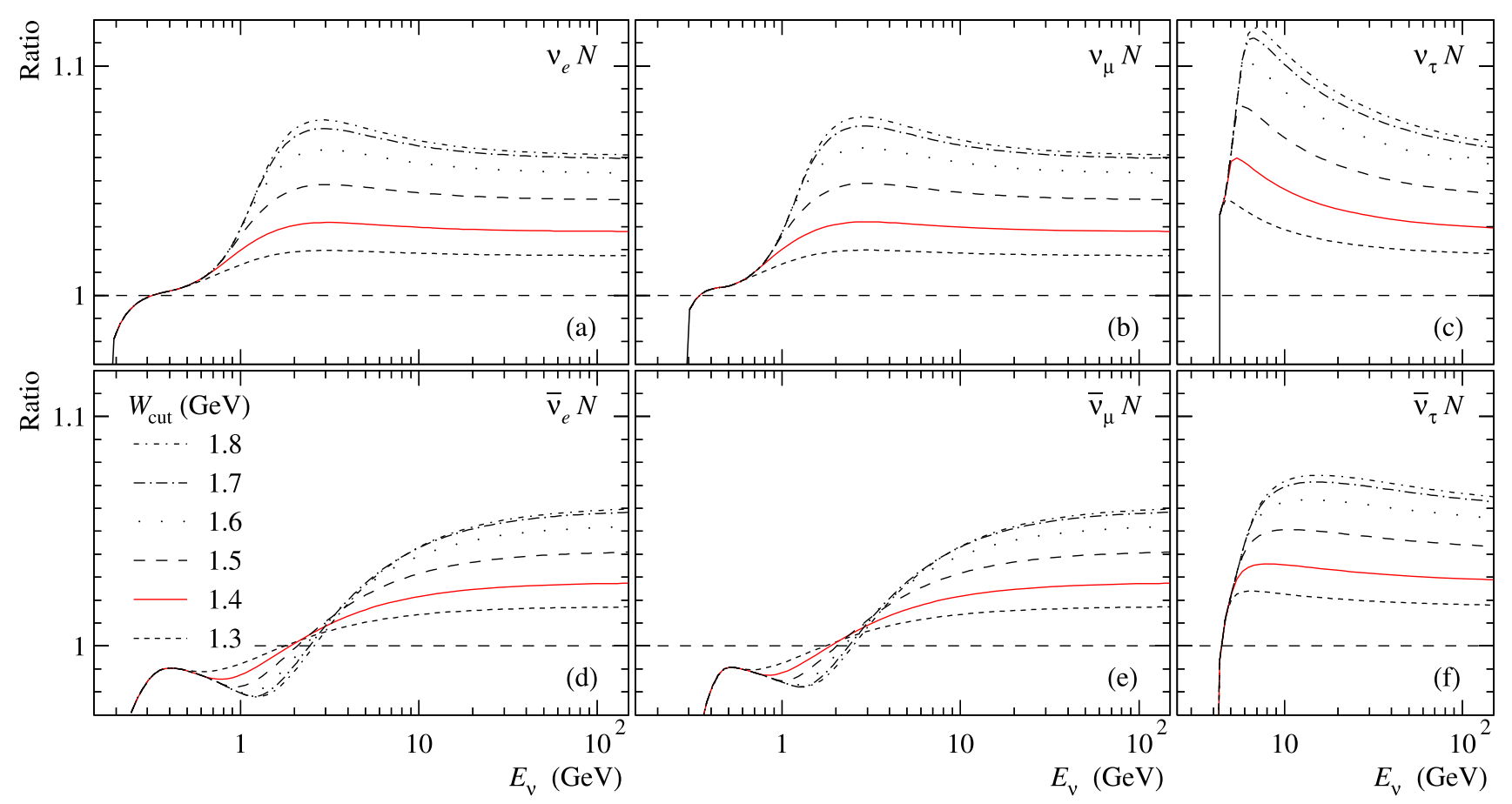

FIG. 1. Ratios of the total cross sections for the resonance $1 \pi$-production reactions predicted with $M_{A}$ obtained from the global fits for $\mathrm{H}_{2}$ and $\mathrm{D}$ data without and with the resonance interference effect as a function of neutrino energy. In order to only illustrate the interference total effect, the cross sections are calculated without NRB contributions for six values of $W$ from $1.3 \mathrm{GeV}$ to $1.8 \mathrm{GeV}$ for the reactions of $\nu_{e}(\mathrm{a}), \nu_{\mu}$ (b), $\bar{\nu}_{\mu}$ (c), $\bar{\nu}_{e}(\mathrm{~d}), \bar{\nu}_{\mu}$ (e), and $\bar{\nu}_{\tau}$ (f) with free isoscalar nucleons.

energies. It should be noted that the cross section calculated with and without taking into account the interference of resonances may require somewhat different values of axial mass for describing the same experimental dataset.

In this study, all the cross sections are calculated with original software package taking into account of interference and using the physical constants published in PDG 2020 [36], whereas the official physical tunes of all GENIE versions are obtained with earlier values of the parameters according to PDG 2016 [37]. Differences between the current and previous values of the central mass, total width, and the branching ratio of resonance reactions with a single pion in the final state have a negligible effect on the cross sections and results of the global fits.

\section{NORMALIZATIONS OF THE BREIT-WIGNER DISTRIBUTIONS}

In all the previous versions of the model (RS, KLN, and KLN-BS) the $W$-dependent Breit-Wigner distributions of resonances are normalized to correction factors, $N_{i}$, which are not well defined. We discuss this problem below and suggest how to avoid them. The normalization factors of the Breit-Wigner distributions, $\eta_{B W}^{i}(W)$, are defined by Eq. (2.33) in [14]

$$
\begin{aligned}
N_{i} & =\int_{W_{\min }}^{\infty} \tilde{\eta}_{B W}^{i}(W) d W, \\
\tilde{\eta}_{B W}^{i}(W) & =\frac{1}{2 \pi} \frac{\Gamma_{i}(W)}{\left(W-M_{i}^{2}\right)^{2}+\Gamma_{i}^{2}(W) / 4}, \\
\Gamma_{i}(W) & =\Gamma_{i}^{0}\left[\frac{q_{\pi}(W)}{q_{\pi}\left(M_{i}\right)}\right]^{2 L+1}, \\
q_{\pi}(W) & =\frac{\sqrt{\left(W^{2}-m_{N^{\prime}}^{2}-m_{\pi}^{2}\right)^{2}-4 m_{N^{\prime}}^{2} m_{\pi}^{2}}}{2 W},
\end{aligned}
$$

where $W_{\min }=m_{N^{\prime}}+m_{\pi}, m_{N^{\prime}}, m_{\pi}, L, M_{i}$, and $\Gamma_{i}^{0}$ are the masses of the final nucleon and pion, total orbital-angular momentum of resonance, resonance mass, and the BreitWigner width, respectively. To calculate $N_{i}$ numerically, we need to define the cutoff for the upper limit of $W$. The result of integration strongly depends on the cutoff value but there are no physical reasons to choose a definite value for it. Figure 2 shows a typical dependence of $\tilde{\eta}_{B W}^{i}(W)$ on the variable $W$ for the $S, P, D$, and $F$ resonance states. Asymptotic behavior of the $S$ resonances is $\sim 1 / W$ unlike the $P, D$, and $F$ resonances. The trivial change of the variable $W=W_{\min } / W^{\prime}$ leads to the limits of integration from 0 to 1 for the expression of $N_{i=S}$. The integrand becomes $\tilde{\eta}_{B W}^{i}\left(W^{\prime}\right) \sim 1 / W^{\prime}$, and its integration gives the nonphysical value for $N_{S}=\infty$. Rein abandoned using the normalization factors of the Breit-Wigner distributions 


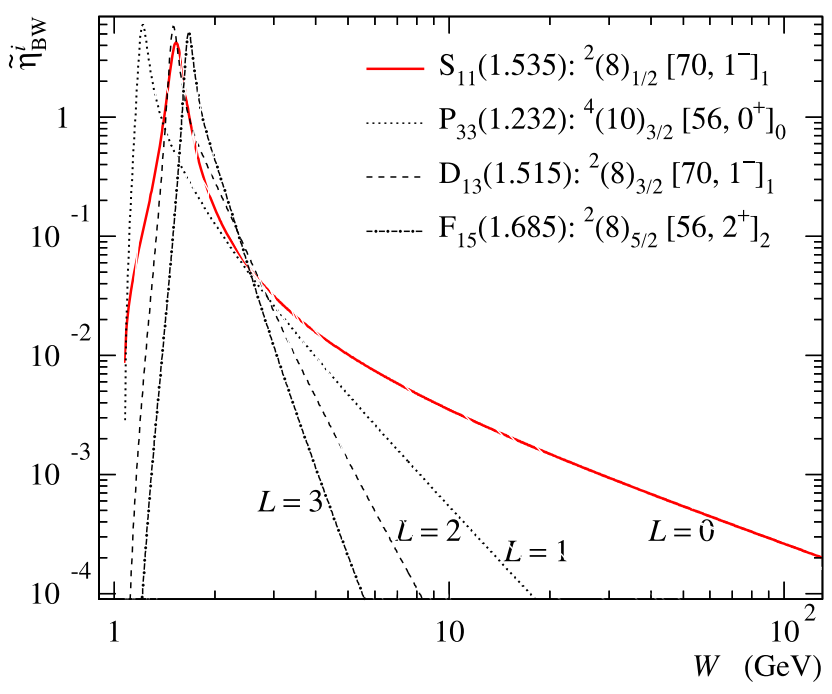

FIG. 2. Breit-Wigner distributions, $\tilde{\eta}_{B W}^{i}(W)$, as functions of the invariant mass of the final hadron system, $W$, for the lightest $S_{11}$ $(L=0), P_{33}(L=1), D_{13}(L=2)$, and $F_{15}(L=3)$ resonance states in the $\nu_{\mu} n \rightarrow \mu^{-} p \pi^{0}$ reaction. The solid line indicates that the $\sim 1 / W$ function leads to unphysical $N_{S}=\infty$.

in [15]. The definition of $N_{i}=1$ is the simplest possibility to avoid the ambiguity in the calculation. Figure 3 shows the ratios of the differential flux-weighted cross sections, $\langle d \sigma / d W\rangle$, for the $\nu_{\mu}$ and $\bar{\nu}_{\mu}$ resonance reactions with the

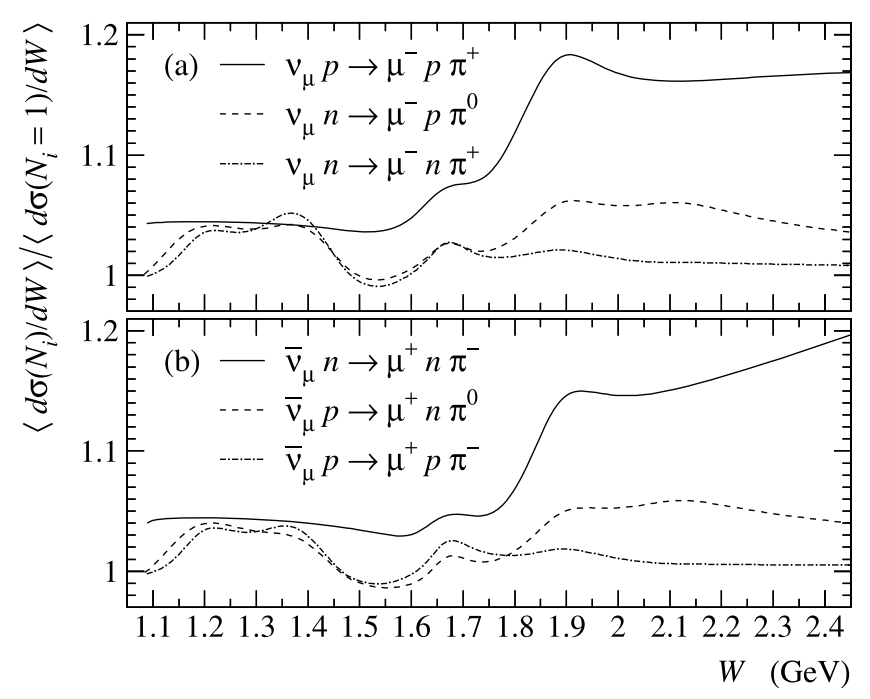

FIG. 3. Ratios of the differential averaged overs the BNL wideband $\nu_{\mu}\left(\bar{\nu}_{\mu}\right)$ beams [39] cross sections as functions of $W$ of the reactions $\nu_{\mu} p \rightarrow \mu^{-} p \pi^{+}, \nu_{\mu} n \rightarrow \mu^{-} p \pi^{0}, \nu_{\mu} n \rightarrow \mu^{-} n \pi^{+}$(a) and $\bar{\nu}_{\mu} n \rightarrow \mu^{+} n \pi^{-}, \bar{\nu}_{\mu} p \rightarrow \mu^{+} n \pi^{0}, \bar{\nu}_{\mu} p \rightarrow \mu^{+} p \pi^{-}$(b) calculated with normalizations of Breit-Wigner factors used in the previous version of KLN-BS model (listed in the notation to the text) to the corresponding cross sections predicted with the definition of $N_{i}=1$. The cross sections are shown for $M_{A}$ and $f_{\mathrm{NRB}}$ obtained from the global fit. values of $N_{i}$ [38] used in the previous version of KLN-BS model to the corresponding cross sections predicted without normalizations. The cross sections are averaged over the energy spectra from the BNL wide-band $\nu_{\mu}$ and $\bar{\nu}_{\mu}$ beams up to $7 \mathrm{GeV}$ [39]. At this energy range the kinematical region of $W$ covers whole the mass range of resonances included in the RS-based models. The greatest effect from the normalizations is achieved at hight values of $W$ and does not significantly affect the total cross sections. The total cross sections calculated with the clear definition of $N_{i}$ are a few percent lower in comparison with ones calculated with ambiguously determined values of normalizations.

The GENIE proposes the ad hoc modification of the Breit-Wigner distributions, $\Gamma^{\prime}(W)$, for the $\Delta$ resonance

$$
\begin{aligned}
& \Gamma^{\prime}(W)=\Gamma_{N \pi} \Gamma(W)+\Gamma_{N \gamma} \Gamma^{0}\left[\frac{1+c / q_{\gamma}(M)}{1+c / q_{\gamma}(W)}\right]^{2} \frac{q_{\gamma}(W)}{q_{\gamma}(M)}, \\
& q_{\gamma}(W)=\frac{W^{2}-m_{N^{\prime}}^{2}}{2 W},
\end{aligned}
$$

where the function $\Gamma(W)$ is defined by the above equation for $L=1, \Gamma_{N \pi}$, and $\Gamma_{N \gamma}$ are the decay-branching ratios of resonance to $N \pi$ and $N \gamma$ states, $M$ is the central value of the mass of the $P_{33}(1232)$ resonance, $c=0.706 \mathrm{GeV}$. The modified $\Gamma^{\prime}(W)$ function is very close to the original $\Gamma(W)$ function because the value of $\Gamma_{N \pi}$ is close to 1 (0.994) and the value of $\Gamma_{N \gamma}$ is close to zero (0.006). In the present calculations we do not use the GENIE modification of the Breit-Wigner function.

\section{METHOD OF DATA FITTING}

We use the following least-square statistical model

$$
\chi^{2}=\sum_{i}\left\{\sum_{j \in G_{i}} \frac{\left[N_{i} T_{i j}(\boldsymbol{\lambda})-E_{i j}\right]^{2}}{\sigma_{i j}^{2}}+\frac{\left(N_{i}-1\right)^{2}}{\sigma_{i}^{2}}\right\} .
$$

In this equation, index $i$ enumerates the experimental data groups $G_{i}$, index $j \in G_{i}$ enumerates the bin-averaged experimental data $E_{i j}$ from the group $G_{i}$, and $\sigma_{i j}$ is the error of $E_{i j}$, without the uncertainty due to the $\nu / \bar{\nu}$ flux normalization. The flux normalization $N_{i}$, individual for each data group $G_{i}$ is treated as a free-fitting parameter and is included into the ordinary penalty term, $\left(N_{i}-1\right)^{2} / \sigma_{i}^{2}$, where $\sigma_{i}$ is the flux normalization error. The $T_{i j}(\lambda)$ represents a bin-averaged theoretical prediction depending on a set of fitting parameters $\lambda$. The procedure of minimization can be simplified by substituting $N_{i}=\mathcal{N}_{i}(\boldsymbol{\lambda})$, into Eq. (1) where $\mathcal{N}_{i}(\lambda)$ are obtained from the analytic solution to the equations $\partial \chi^{2} / \partial N_{i}=0$,

$$
\mathcal{N}_{i}(\lambda)=\frac{1+\sigma_{i}^{2} \sum_{j \in G_{i}} \sigma_{i j}^{-2} T_{i j}(\lambda) E_{i j}}{1+\sigma_{i}^{2} \sum_{j \in G_{i}} \sigma_{i j}^{-2} T_{i j}^{2}(\lambda)} .
$$


As it follows from the analysis, the deviation of the normalization factors $N_{i}$ from unity for each data group $G_{i}$ does not exceed the doubled experimental uncertainty of the corresponding $\nu_{\mu}$ or $\bar{\nu}_{\mu}$ flux normalization.

All the fits are done with the CERN function minimization and error analysis package MINUIT (version 94.1) $[40,41]$, thus taking care of getting an accurate error matrix. The errors of the output parameters quoted below correspond to the usual one and two-standard deviation $(1 \sigma$ and $2 \sigma)$ errors. Numerical calculations with GENIE are done with the JINR cloud infrastructure of Multifunctional Information and Computing Complex [42].

\section{EXPERIMENTAL DATASET}

We analyzed and classified all available $1 \pi$-production experimental data, presented as the $\nu_{\mu}$ and $\bar{\nu}_{\mu}$ scattering cross sections on hydrogen and deuterium targets measured in ANL [43-53], BNL [54-58], FNAL [59-62], and CERN [63-80] accelerator experiments from the beginning of the 1970s to the 1990s with energy ranges from about hundreds of $\mathrm{MeV}$ (ANL) to tens of $\mathrm{GeV}$ (CERN).

The most statistically reliable measurements of the total and differential cross sections, which were not superseded or reconsidered (due to increased statistics, revised of neutrino fluxes, etc.) in the subsequent reports of the same experimental groups, have been selected for our analysis. Further information and reanalysis of the original data are taken from the papers [81-85].

In the statistical analysis we use the experimental data on measuring the ratio of the total cross section of the resonance $1 \pi^{+}$-production reactions to the total cross section of the $\nu_{\mu} n \rightarrow \mu^{-} p$ reaction. The CC QES interactions on a bound nucleon in any kind of nuclei are calculated according to the phenomenological prescription of the effective running quasielastic axial-vector mass, $M_{A}^{\text {run }}$ (see [86] and references therein). The parameters of $M_{A}^{\text {run }}$ are obtained from the global fit to all available data on the $\nu_{\mu}$ and $\bar{\nu}_{\mu}$ cross sections of CC QES reactions. For neutrino reactions with deuterium and hydrogen, the running axial-vector mass is reduced to the current axial-vector mass of $M_{A}^{\mathrm{QES}}=1.008 \pm$ $0.025(0.029) \mathrm{GeV}$.

The process $\nu_{\mu} p \rightarrow \mu^{-} \Delta^{++}$was studied in experiments with deuterium targets at ANL 1979 [50] (nine data points), BNL 1986 [58] (8), and CERN 1980 [74] (7) with the cuts of $W<1.4 \mathrm{GeV}$. The data are presented as 15 and 9 data points for the total and flux-averaged
$Q^{2}$-dependent differential cross sections, respectively. The total and differential cross sections of the $\nu_{\mu} p \rightarrow$ $\mu^{-} \Delta^{++}$reaction were obtained in ANL 1979 [50] experiment. We use the differential cross sections for the global fit and the total cross sections for the comparison only. The experimental data of the total cross sections of the reaction $\Delta^{++}$resonance production were measured at ANL 1973 [43] and FNAL 1978 [60,61] and were obtained with no experimental cuts for $W$. The cross sections measured at FNAL 1978 [60,61] are corrected by a factor of 1.1 due to the following reason. The particle fluxes from the broad-band horn beams at FNAL were not measured directly. Therefore, the results were normalized by obtaining the ratio $\nu_{\mu} p \rightarrow \mu^{-} \Delta^{++}$to all $\mathrm{CC}$ events and using the total cross section from FNAL 1977 [87] and CERN BEBC 1977 [88] parametrized as $\sigma(\nu N)=\left(0.77-0.085 \lg E_{\nu}\right) E_{\nu} \times 10^{-38} \mathrm{~cm}^{2}$. This parametrization underestimates the cross section to about $11 \%$ (the estimate is made with the GRV98 [89] parton distribution functions model). Actually, the papers $[43,60,61,69]$ do not contain clear information about of the limits for $W$. It is not obvious that the old experiments with small statistics of the neutrino-induced events could reliably identify the $\Delta^{++}$resonance and background from other resonances. If the data of ANL 1973 [43] and FNAL 1978 [60,61] experiments were obtained for $W<1.4 \mathrm{GeV}$, the results of their measurement would be superseded in the subsequent papers. To avoid misleading interpretation of the data, we do not use these datasets for the global fits.

The experimental data for the cross sections of the $\nu_{\mu} p \rightarrow \mu^{-} p \pi^{+}$reaction are obtained at ANL 1982 [53] (23 data points), BNL 1986 [58] (7), FNAL 1978 [60,61] (2), and CERN BEBC 1984 [76] (6), 1986 [77] (9), and 1990 [80] (6), on deuterium targets for different values of the experimental cuts for $W$. The experiments to study the $1 \pi$-production reactions at ANL accelerator center were terminated in 1982 and not resumed, but the data accumulated by ANL 1982 experiment [53] are still the most important. The total cross sections were measured with cuts for $W$ of $1.4,1.6 \mathrm{GeV}$ and with no cut for $W$. The total cross sections measured for $W<1.4 \mathrm{GeV}$ and with no cut for $W$ were revised in the paper [84]. For the global fit we use the revised total cross sections with no cut for $W$. Instead of the total cross sections measured for $W<1.4 \mathrm{GeV}$ we include into the global fit the original flux-averaged $Q^{2}$-dependent cross sections. For the global fits, we use 16 data points recalculated from the number of events measured at ANL 1982 [53] (7 data points) and BNL 1986 [58] (9) to the total cross sections ratio of the 
$\nu_{\mu} p \rightarrow \mu^{-} p \pi^{+}$and CC QES reactions [81-83]. In the experiment with the deuterium target CERN BEBC 1990 [80], the flux-averaged $Q^{2}$-dependent differential cross sections were studied for special cuts for $W$. Unfortunately, the differential cross sections cannot be used in the global fits because the original $\nu_{\mu}$ flux is unknown. The data measured at ANL [46,47,49,50], FNAL [59,62], and CERN [78] are obsolete. The dataset consists of 69 data points satisfying our selection criteria of the experimental data.

The data on the measurement of the total cross sections of the $\bar{\nu}_{\mu} n \rightarrow \mu^{+} n \pi^{-}$reaction were obtained at CERN BEBC 1983 [75] (6 data points) and 1990 [80] (6). The paper with preliminary results of the experiment [75] contains the values of the total cross sections for the $W$ cuts of 1.4 and $2 \mathrm{GeV}$, while the final paper provides the data only for $W<2 \mathrm{GeV}$. We use the final and preliminary data for $W<2 \mathrm{GeV}$ but not reconsidered data for $W<1.4 \mathrm{GeV}, 12$ data points in total. As well as in the case of the $\nu_{\mu} p \rightarrow \mu^{-} p \pi^{+}$reaction, we cannot use the data on the flux-averaged cross sections measured at CERN BEBC 1990 [80]. Thus, 12 data points are included into the global fit.

The cross sections of the $\nu_{\mu} n \rightarrow \mu^{-} p \pi^{0}$ reaction were measured at ANL 1982 [53] (16 data points), BNL 1986 [58] (10), and CERN BEBC 1984 [76] (6) with hydrogen and deuterium targets for the cuts of $W<1.4,1.6$, $2.0 \mathrm{GeV}$ and without a cut. The total cross sections measured at ANL [53] and BNL 1986[58] for the cut of $W<1.4 \mathrm{GeV}$ and without a cut were revised by Rodrigues et al. [84]. The total cross sections measured at CERN BEBC 1990 [80] for the cut of $W<2 \mathrm{GeV}$ were revised by Hawker [85]. In the global fit we use the revised data. The raw data on measuring the $Q^{2}$-distributions obtained at ANL [53] and BNL [58] with no cut for $W$ were recalculated by Furuno et al. [81-83] to the ratio of the total cross sections of the $\nu_{\mu} n \rightarrow \mu^{-} p \pi^{0}$ to CC QES reactions. We include these 13 data points into the global fit. We cannot use the data on the fluxaveraged cross sections measured at CERN BEBC 1990 [80] for the same reason as in the case of the $\nu_{\mu} p \rightarrow$ $\mu^{-} p \pi^{+}$and $\bar{\nu}_{\mu} n \rightarrow \mu^{+} n \pi^{-}$reactions. The data measured at ANL [45,47,49,50] and CERN GGM 1977 [68] are updated in recent publications of the same author groups. Thus, there are 45 data points suitable for the global fit.

The cross sections of the $\nu_{\mu} n \rightarrow \mu^{-} n \pi^{+}$reaction were measured at ANL 1982 [53] (16 data points), BNL 1986 [58] (11), and CERN BEBC 1984 [76] (6). The same author groups revised the original data (except for the data measured at CERN BEBC [80] for $W<2 \mathrm{GeV}$ ).
Hence, there are 33 data points for the total cross sections and 14 data points for the total cross section ratios of the $\nu_{\mu} n \rightarrow \mu^{-} n \pi^{+}$to CC QES reactions [81-83].

The cross sections of the $\bar{\nu}_{\mu} p \rightarrow \mu^{+} p \pi^{-}$reaction were measured at FNAL 1980 [62], and CERN BEBC 1983 [75] (6 data points), 1986 [77] (4), and 1990 [80] (6). Therefore, 16 data points can be used for global fits.

We use six data points for the total cross section sums of the $\nu_{\mu} p \rightarrow \mu^{-} p \pi^{+}$and $\bar{\nu}_{\mu} p \rightarrow \mu^{+} p \pi^{-}$reactions measured at CERN BEBC 1990 [80]. The cross sections of the $\nu_{\mu} n \rightarrow$ $\mu^{-} p \pi^{0}$ and $\nu_{\mu} n \rightarrow \mu^{-} n \pi^{+}$reactions with a neutral particle in the final state cannot be analyzed in the same experiment with kinematic fitting and were recognized by topology of secondary particles. The problem of the event separation is more difficult than any others because the selection is estimated for proton or pion tracks at the technical limit of effective bubble density. This special feature leads to ambiguity in the interpretation of a specific reaction. Due to this reason six data points are not included into the global fit.

The data of flux-averaged $Q^{2}$ - and $W$-distributions measured at ANL 1980 [51], 1982 [53], BNL 1980 [55], 1986 [58], FNAL 1980 [62], BEBC 1986 [77], 1990 [80], are used only for comparison and not for global fits. Absolute values and shape of the distributions as well as the flux-averaged differential cross sections depending on $Q^{2}$ are primarily determined by the shape of neutrino fluxes used in data processing by the authors of the experiments. The differential distribution estimated at mean energy of the neutrino flux or calculated with a nonoriginal flux provides only a qualitative comparison with the measured distribution, i.e., approximate calculations of differential distributions are a source of uncontrolled systematic uncertainty for the results of the global fits.

We avoid using the differential cross sections measured at CERN BEBC 1990 [80] because the data points seem self-contradictory. The shapes of the cross sections for different reactions are inconsistent with the predictions by the RS approach. These data lead to an increase in $\chi^{2}$ values but do not to clarify the fitted parameters. The data of this experiment are shown for a qualitative comparison only.

There are no available experimental data for the total or differential cross sections of the $\bar{\nu}_{\mu} p \rightarrow \mu^{+} n \pi^{0}$ reaction measured on hydrogen or deuterium targets.

\section{RESULTS OF THE GLOBAL FITS}

In our previous study [20], the value of $M_{A}$ was obtained from the global likelihood analysis for total 
cross sections measured at ANL 1982 [53], BNL 1986 [58], FNAL 1978 [61], 1980 [62], CERN GGM 1978 [68,69], 1979 [73], CERN BEBC 1980 [74], 1983 [75], 1986 [77], 1990 [80], and IHEP SKAT [90]. The dataset included cross sections of the reactions measured on various nuclear targets with and without NRB contribution according to the RS model. That dataset contained 190 data points with 127 data points of the $\nu_{\mu}$ cross sections (66.84\% of the total number) and 63 data points of the $\bar{\nu}_{\mu}$ cross sections $(33.16 \%)$. In the previous analysis we did not run a likelihood tune of NRB and used the values of the Breit-Wigner normalizations calculated numerically according to the formal definition. Also, the normalizations of the experimental data are not discussed.

The value of the resonance axial-vector mass

$$
M_{A}=1.18_{-0.07(0.08)}^{+0.07(0.09)} \mathrm{GeV}
$$

was obtained with $\chi^{2} / \mathrm{ndf}=186.6 /(105-11) \approx 1.99$ from the global fit for the cross sections of the $\nu_{\mu} p \rightarrow \mu^{-} \Delta^{++}, \nu_{\mu} p \rightarrow \mu^{-} p \pi^{+}$, and $\bar{\nu}_{\mu} n \rightarrow \mu^{+} n \pi^{-}$reactions which do not require NRB in the RS approach and are measured in experiments on hydrogen and deuterium targets only. The dataset consists of 105 experimental data points with 93 data points of the $\nu_{\mu}$ cross sections (88.6\% of the total number) and 12 data points of the $\bar{\nu}_{\mu}$ cross sections $(11.4 \%)$.

The value of the adjustable parameter

$$
f_{\mathrm{NRB}}=1.12 \pm 0.10(0.13)
$$

was obtained with $\chi^{2} / \mathrm{ndf}=428.4 /(114-8) \approx 4.04$ from the fit for the cross sections of the $\nu_{\mu} n \rightarrow \mu^{-} p \pi^{0}$, $\nu_{\mu} n \rightarrow \mu^{-} n \pi^{+}$, and $\bar{\nu}_{\mu} p \rightarrow \mu^{+} p \pi^{-}$reactions which require the consideration of NRB in the RS approach. The dataset consists of 114 experimental data points with 92 points of the $\nu_{\mu}$ cross sections ( $81 \%$ of the total number), 16 data points of the $\bar{\nu}_{\mu}$ cross sections (14\%), and 6 data points for sum of the $\nu_{\mu}$ and $\bar{\nu}_{\mu}$ cross sections $(5 \%)$.

TABLE I. Experimental datasets and data tapes involved into the global fit of $M_{A}$, results of individual fits for $M_{A}$ of selected datasets, normalization factors $N_{\nu, \bar{\nu}}$, absolute and normalized to ndf values of $\chi^{2}$ (only absolute value in the case of ndf $=0$ ), where ndf $=\mathrm{NP}-N_{p}$, NP is the number of experimental data bins in the dataset, and $N_{p}=1,2$ is the number of free parameters. Statistical errors of the fitted parameters correspond to one and two (shown in brackets) standard deviations. The datasets of ANL 1982 [53] and

\begin{tabular}{|c|c|c|c|c|}
\hline Experiment & Data & $M_{A}(\mathrm{GeV})$ & $N_{\nu, \bar{\nu}}$ & $\chi^{2} / \mathrm{ndf}$ \\
\hline Barish et al., ANL 1979 [50] & $\left\langle d \sigma\left(\nu_{\mu} p \rightarrow \mu^{-} \Delta^{++}\right) / d Q^{2}\right\rangle$ & $1.03_{-0.13(0.20)}^{+0.15(0.26)}$ & $1.09_{-0.13(0.22)}^{+0.14(0.22)}$ & $7.27 / 7=1.04$ \\
\hline \multirow[t]{2}{*}{ Radecky et al., ANL 1982 [53] } & $\sigma\left(\nu_{\mu} p \rightarrow \mu^{-} p \pi^{+}\right)$ & $1.16_{-0.06(0.10)}^{+0.07(0.11)}$ & $1.04_{-0.04(0.07)}^{+0.04(0.07)}$ & $90.19 / 21=4.29$ \\
\hline & $\sigma\left(\nu_{\mu} p \rightarrow \mu^{-} p \pi^{+}\right) / \sigma^{\mathrm{QES}}$ & $1.13_{-0.09(0.17)}^{+0.09(0.18)}$ & 1 & $12.93 / 6=2.16$ \\
\hline \multirow[t]{2}{*}{ Kitagaki et al., BNL 1986 [58] } & $\sigma\left(\nu_{\mu} p \rightarrow \mu^{-} \Delta^{++}\right) \& \sigma\left(\nu_{\mu} p \rightarrow \mu^{-} p \pi^{+}\right)$ & $1.20_{-0.12(0.18)}^{+0.14(0.24)}$ & $1.07_{-0.11(0.18)}^{+0.12(0.20)}$ & \\
\hline & $\sigma\left(\nu_{\mu} p \rightarrow \mu^{-} p \pi^{+}\right) / \sigma^{\mathrm{QES}}$ & $1.25_{-0.03(0.06)}^{+0.03(0.06)}$ & 1 & $23.60 / 8=2.95$ \\
\hline Bell et al., FNAL $1978[60,61]$ & $\sigma\left(\nu_{\mu} p \rightarrow \mu^{-} p \pi^{+}\right)$ & $1.11_{-0.18(0.29)}^{+0.20(0.33)}$ & $1.00_{-0.16(0.26)}^{+0.16(0.26)}$ & 0.02 \\
\hline Allen et al., CERN BEBC 1980 [74] & $\sigma\left(\nu_{\mu} p \rightarrow \mu^{-} \Delta^{++}\right)$ & $1.09_{-0.13(0.20)}^{+0.15(0.25)}$ & $1.00_{-0.15(0.24)}^{+0.15(0.24)}$ & $6.54 / 5=1.31$ \\
\hline Allasia et al., CERN BEBC 1983 [75] & $\sigma\left(\bar{\nu}_{\mu} n \rightarrow \mu^{+} n \pi^{-}\right)$ & $0.98_{-0.15(0.24)}^{+0.17(0.28)}$ & $1.00_{-0.15(0.25)}^{+0.15(0.25)}$ & $2.24 / 4=0.56$ \\
\hline Barlag et al., CERN BEBC 1984 [76] & $\sigma\left(\nu_{\mu} p \rightarrow \mu^{-} p \pi^{+}\right)$ & $1.10_{-0.14(0.22)}^{+0.16(0.27)}$ & $1.00_{-0.15(0.24)}^{+0.15(0.25)}$ & $1.36 / 4=0.34$ \\
\hline \multirow[t]{2}{*}{ Allen et al., CERN BEBC 1986 [77] } & $\sigma\left(\nu_{\mu} p \rightarrow \mu^{-} p \pi^{+}\right), W<2 \mathrm{GeV}$ & $1.07_{-0.13(0.20)}^{+0.16(0.26)}$ & $1.00_{-0.15(0.24)}^{+0.15(0.24)}$ & $4.38 / 3=1.46$ \\
\hline & $\sigma\left(\nu_{\mu} p \rightarrow \mu^{-} p \pi^{+}\right), W>2 \mathrm{GeV}$ & $0.98_{-0.19(0.31)}^{+0.19(0.33)}$ & $1.00_{-0.15(0.25)}^{+0.15(0.25)}$ & $3.84 / 2=1.92$ \\
\hline \multirow[t]{2}{*}{ Allasia et al., CERN BEBC 1990 [80] } & $\sigma\left(\nu_{\mu} p \rightarrow \mu^{-} p \pi^{+}\right)$ & $1.15_{-0.11(0.18)}^{+0.13(0.22)}$ & $1.00_{-0.13(0.21)}^{+0.13(0.21)}$ & $3.69 / 4=0.92$ \\
\hline & $\sigma\left(\bar{\nu}_{\mu} n \rightarrow \mu^{+} n \pi^{-}\right)$ & $1.29_{-0.12(0.18)}^{+0.14(0.23)}$ & $1.00_{-0.13(0.21)}^{+0.13(0.21)}$ & $2.29 / 4=0.57$ \\
\hline
\end{tabular}
BNL 1986 [58] include the original data and the data recalculated by Furuno et al. [81-83] and Rodrigues et al. [84]. 
To illustrate the effect of normalizations of the BreitWigner distributions on the discussed tunable parameters, we perform global fits using a more traditional choice of normalizations according to the previous version of KLN-BS model: $M_{A}=1.12 \pm 0.06(0.08) \mathrm{GeV}$ with $\chi^{2} / \mathrm{ndf}=201.2 /(105-11) \approx 2.12$ and $f_{\mathrm{NRB}}=1.09 \pm$ $0.11(0.13)$ with $\chi^{2} /$ ndf $=413.1 /(114-8) \approx 3.90$. The parameters extracted from the new global and test fits as well as $\chi^{2}$-values are slightly different, which means that normalizations of distributions do not significantly affect the global fit for the model parameters. However, unreliable/unphysical constants should be avoided.

Tables I and II collect the values of $M_{A}$ and the adjustable parameter $f_{\mathrm{NRB}}$ with the normalization factors obtained from the individual fits for each experimental datasets involved into the global fit. The values of the parameters for individual fits agree with each other within the errors.
Figures 4-8 show the experimental data in comparison with the predicted cross sections for default values for $M_{A}$ and $f_{\mathrm{NRB}}$ parameters. In all figures the data points marked with open symbols are not included into the statistical analysis as well as the averaged cross sections shown with hatched rectangles. The experimental data are not multiplied by normalization factors obtained from the global fits. We do not include into the global fit the experimental data on the flux-weighted total cross sections shown in Figs. 4(b), 5(a), and 7(e), 7(d) as shaded areas. The ANL and BNL data measured for the $\nu_{\mu} p \rightarrow$ $\mu^{-} p \pi^{+}$reaction without cut for $W$ were corrected for nuclear effects and FSI [91]. The global fit with the corrected data leads to the same $M_{A}$. The point is that the cross section weakly depends on the axial mass in the low neutrino energy range and corrections don't significantly change the data.

TABLE II. The same items as in Table I but for the datasets involved into the individual and global fits of $f_{\text {NRB }}$. The data recalculated by Furuno et al. [81-83] and Rodrigues et al. [84] marked by indices ${ }^{\mathrm{F}}$ and ${ }^{\mathrm{R}}$, respectively.

\begin{tabular}{|c|c|c|c|c|}
\hline Experiment & Data & $f_{\mathrm{NRB}}$ & $N_{\nu, \bar{\nu}}$ & $\chi^{2} / \mathrm{ndf}$ \\
\hline \multirow[t]{4}{*}{ Radecky et al., ANL 1982 [53] } & $\begin{array}{c}\sigma\left(\nu_{\mu} n \rightarrow \mu^{-} p \pi^{0}\right) \& \sigma\left(\nu_{\mu} n \rightarrow \mu^{-} n \pi^{+}\right) \\
W<1.6 \mathrm{GeV}\end{array}$ & $1.24_{-0.22(0.38)}^{+0.19(0.31)}$ & $1.00_{-0.05(0.09)}^{+0.05(0.09)}$ & $16.20 / 6=2.70$ \\
\hline & $\begin{array}{c}\sigma\left(\nu_{\mu} n \rightarrow \mu^{-} p \pi^{0}\right) \& \sigma\left(\nu_{\mu} n \rightarrow \mu^{-} n \pi^{+}\right) \\
W<1.4 \mathrm{GeV}, \text { no cut } \\
\text { R }\end{array}$ & $1.40_{-0.14(0.24)}^{+0.13(0.21)}$ & $1.00_{-0.04(0.06)}^{+0.04(0.06)}$ & $69.48 / 22=3.16$ \\
\hline & 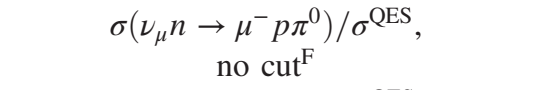 & $1.21_{-0.46(-)}^{+0.33(0.59)}$ & 1 & $9.59 / 3=3.20$ \\
\hline & $\begin{array}{c}\sigma\left(\nu_{\mu} n \rightarrow \mu^{-} n \pi^{+}\right) / \sigma^{\mathrm{QES}}, \\
{\text { no } \operatorname{cut}^{\mathrm{F}}}\end{array}$ & $1.14_{-0.19(0.43)}^{+0.17(0.31)}$ & 1 & $13.03 / 4=3.26$ \\
\hline \multirow[t]{4}{*}{ Kitagaki etal., BNL 1986 [58] } & $\begin{array}{c}\sigma\left(\nu_{\mu} n \rightarrow \mu^{-} p \pi^{0}\right) \\
\text { no cut }\end{array}$ & $1.29_{-0.42(0.74)}^{+0.42(0.71)}$ & $1.10_{-0.19(0.30)}^{+0.19(0.31)}$ & $28.17 / 8=3.52$ \\
\hline & $\begin{array}{c}\sigma\left(\nu_{\mu} n \rightarrow \mu^{-} n \pi^{+}\right) \\
\text {no cut }\end{array}$ & $1.00_{-0.27(0.45)}^{+0.30(0.52)}$ & $0.99_{-0.20(0.32)}^{+0.20(0.32)}$ & $44.81 / 9=4.98$ \\
\hline & 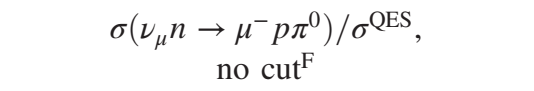 & $1.61_{-0.10(0.19)}^{+0.09(0.17)}$ & 1 & $25.82 / 8=3.23$ \\
\hline & $\begin{array}{c}\sigma\left(\nu_{\mu} n \rightarrow \mu^{-} n \pi^{+}\right) / \sigma^{\mathrm{QES}} \\
{\operatorname{no~} \mathrm{cut}^{\mathrm{F}}}^{\mathrm{F}}\end{array}$ & $0.91_{-0.08(0.16)}^{+0.07(0.13)}$ & 1 & $58.03 / 8=7.25$ \\
\hline Allasia et al., CERN BEBC 1983 [75] & $\begin{array}{c}\sigma\left(\bar{\nu}_{\mu} p \rightarrow \mu^{+} p \pi^{-}\right) \\
W<2 \mathrm{GeV}\end{array}$ & $1.18_{-0.31(0.54)}^{+0.29(0.48)}$ & $1.00_{-0.15(0.25)}^{+0.15(0.24)}$ & $6.53 / 4=1.63$ \\
\hline Barlag et al., CERN BEBC 1984 [76] & $\begin{array}{c}\sigma\left(\nu_{\mu} n \rightarrow \mu^{-} p \pi^{0}\right) \& \sigma\left(\nu_{\mu} n \rightarrow \mu^{-} n \pi^{+}\right) \\
W<2 \mathrm{GeV}\end{array}$ & $0.72_{-0.21(0.37)}^{+0.19(0.30)}$ & $0.92_{-0.10(0.16)}^{+0.10(0.16)}$ & $33.01 / 10=3.30$ \\
\hline \multirow[t]{2}{*}{ Allen et al., CERN BEBC 1986 [77] } & $\begin{array}{c}\sigma\left(\bar{\nu}_{\mu} p \rightarrow \mu^{+} p \pi^{-}\right) \\
W<2 \mathrm{GeV}\end{array}$ & $0.25 \pm 100 \%$ & $1.00_{-0.15(0.25)}^{+0.12(0.18)}$ & $0.86 / 3=0.29$ \\
\hline & $\begin{array}{c}\sigma\left(\bar{\nu}_{\mu} p \rightarrow \mu^{+} p \pi^{-}\right) \\
W>2 \mathrm{GeV}\end{array}$ & $0.88_{-0.19(0.32)}^{+0.18(0.30)}$ & $1.01_{-0.15(0.23)}^{+0.15(0.23)}$ & $2.45 / 2=1.22$ \\
\hline \multirow[t]{2}{*}{ Allasia et al., CERN BEBC 1990 [80] } & $\begin{array}{c}\sigma\left(\bar{\nu}_{\mu} p \rightarrow \mu^{+} p \pi^{-}\right) \\
W<2 \mathrm{GeV}\end{array}$ & $1.30_{-0.20(0.31)}^{+0.22(0.38)}$ & $1.00_{-0.15(0.25)}^{+0.15(0.25)}$ & $10.84 / 4=2.71$ \\
\hline & $\begin{array}{c}\sigma\left(\nu_{\mu} n \rightarrow \mu^{-} n \pi^{+}\right)+\sigma\left(\bar{\nu}_{\mu} p \rightarrow \mu^{+} p \pi^{-}\right) \\
W>2 \mathrm{GeV}\end{array}$ & $1.10_{-0.14(0.36)}^{+0.23(0.37)}$ & $\begin{array}{l}0.85 \\
0.99\end{array}$ & $27.12 / 3=9.04$ \\
\hline
\end{tabular}




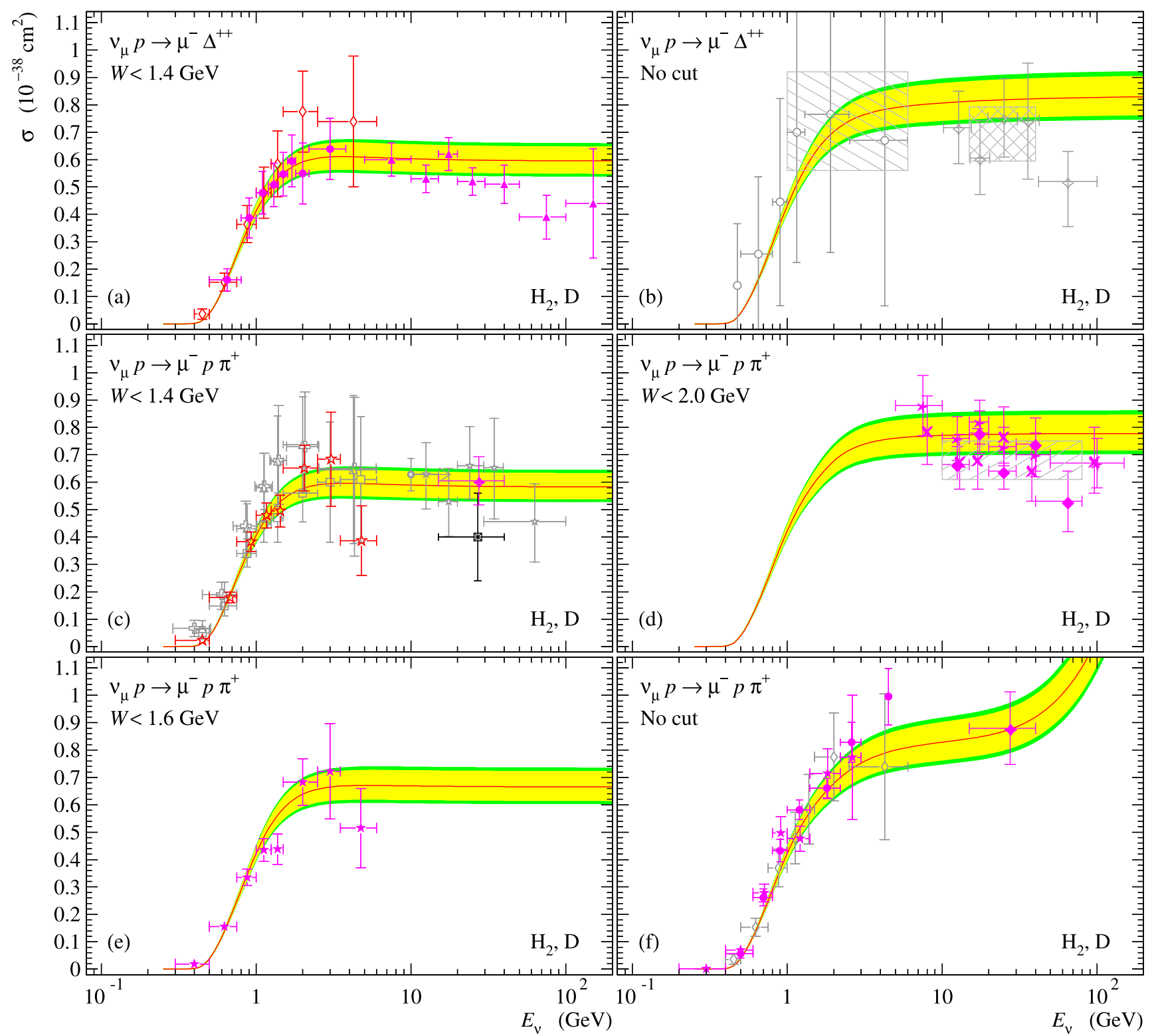

\begin{tabular}{|c|c|c|}
\hline Campbell et al., ANL 1973 & 许 $\star$ Radecky et al., ANL 1982 & A Allen et al., CERN BEBC 1980 \\
\hline$\rightsquigarrow$ Barish et al., ANL 1975 & - Kitagaki et al., BNL 1986 & x Barlag et al., CERN BEBC 1984 \\
\hline$\triangle$ Barish et al., ANL 1976 & Bell et al., FNAL 1978 & Allen et al., CERN BEBC 1986 \\
\hline$\square$ Barnes et al., ANL 1978 & 곡 et al., FNAL 1978 & ○ Jones et al., CERN BEBC 1989 \\
\hline$\diamond \diamond$ Barish et al., ANL 1979 & 田 Barish et al., FNAL 1980 & * Allasia et al., CERN BEBC 1990 \\
\hline
\end{tabular}

FIG. 4. Total cross sections of the reactions $\nu_{\mu} p \rightarrow \mu^{-} \Delta^{++}$(a), (b) and $\nu_{\mu} p \rightarrow \mu^{-} p \pi^{+}$(c)-(f) as functions of neutrino energy predicted with $M_{A}$ obtained from the global fit for $\mathrm{H}_{2}$ and D data in comparison with the experimental data measured at ANL 1973 [43], 1975 [46], 1976 [47], 1978 [49], 1979 [50], 1982 [53], BNL 1986 [58], FNAL 1978 [59,60], 1980 [62], and CERN BEBC 1980 [74], 1984 [76], 1986 [77], 1989 [78], 1990 [80]. The original data of ANL 1982 [53] and BNL 1986 [58] are recalculated by Rodrigues et al. [84]. Error bars of the experimental data points show the statistical and systematical errors added quadratically with no errors of $\nu_{\mu}$ flux normalizations. Inner and outer shaded bands around the curves demonstrate the uncertainty of the fitted value of $M_{A}$ corresponding to $1 \sigma$ and $2 \sigma$ errors, respectively. Titles of the reactions, targets, and experimental cuts on $W$ are given in the legends in each panel. 

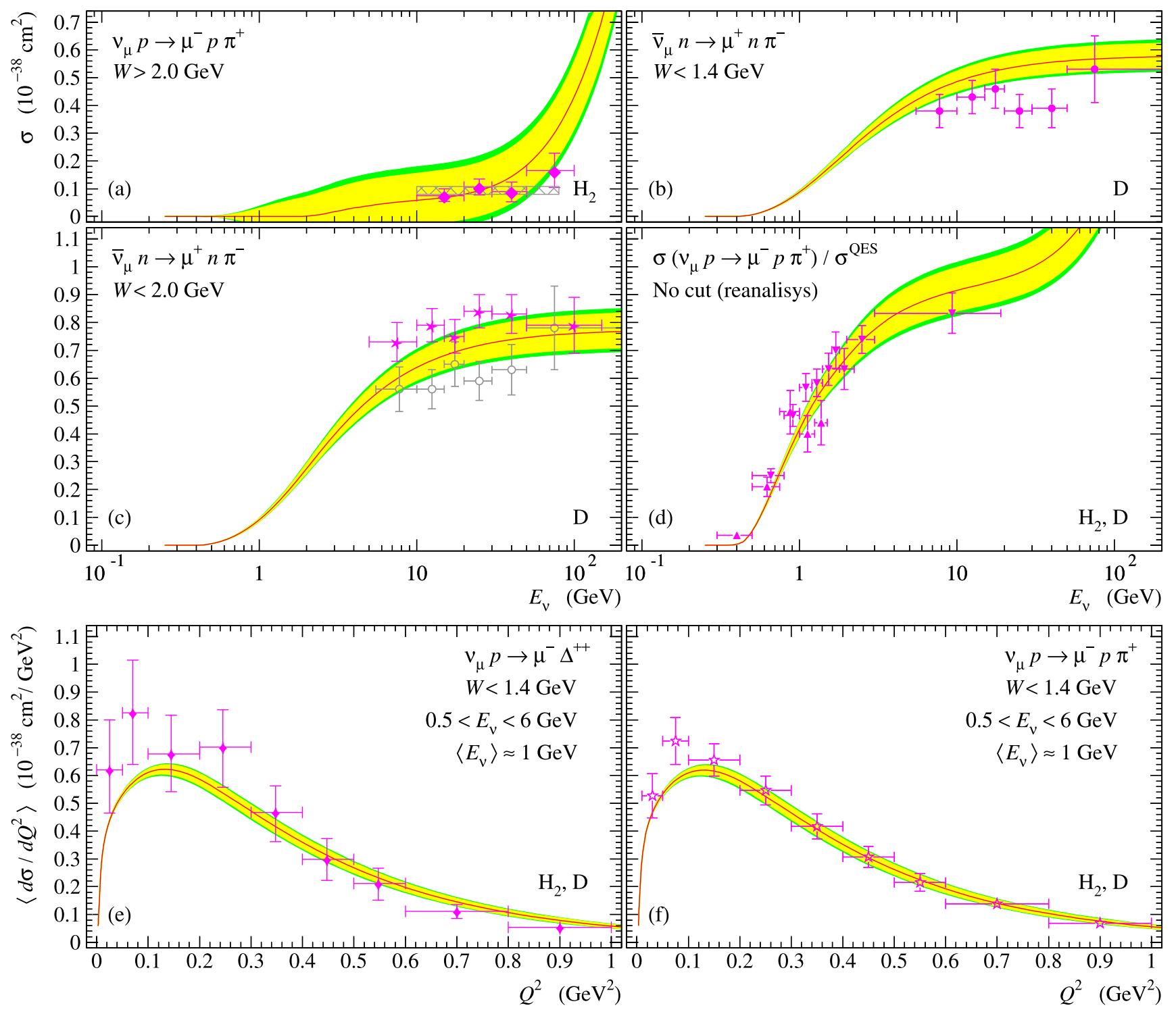

Barish et al., ANL 1979

औ $\star$ Radecky et al., ANL 1982

Furuno et al., ANL 2003 v Furuno et al., BNL 2003

* Allasia et al., CERN BEBC 1990
- $\quad$ Allasia et al., CERN BEBC 1983

- Allen et al., CERN BEBC 1986

FIG. 5. Total cross sections of the reaction $\nu_{\mu} p \rightarrow \mu^{-} p \pi^{+}$(a), $\bar{\nu}_{\mu} n \rightarrow \mu^{+} n \pi^{-}$(b), (c), ratio of the total cross sections of the reaction $\nu_{\mu} p \rightarrow \mu^{-} p \pi^{+}$to the quasielastic reaction $\nu_{\mu} n \rightarrow \mu^{-} p(\mathrm{~d})$ as functions of neutrino energy and $Q^{2}$-dependent flux-weighted differential cross sections of the reactions $\nu_{\mu} p \rightarrow \mu^{-} \Delta^{++}$(e) and $\nu_{\mu} p \rightarrow \mu^{-} p \pi^{+}$(f) predicted with $M_{A}$ obtained from the global fit for $\mathrm{H}_{2}$ and $\mathrm{D}$ data in comparison with the experimental data measured at ANL 1979 [50], 1982 [53] and CERN BEBC 1983 [75], 1986 [77], 1990 [80]. Differential cross sections are averaged over the flux borrowed from [48]. Titles of the reactions, targets, experimental cuts on $W$, and additional information are given in the legends. Notation for the solid curves and shaded bands is the same as in Fig. 4 . 


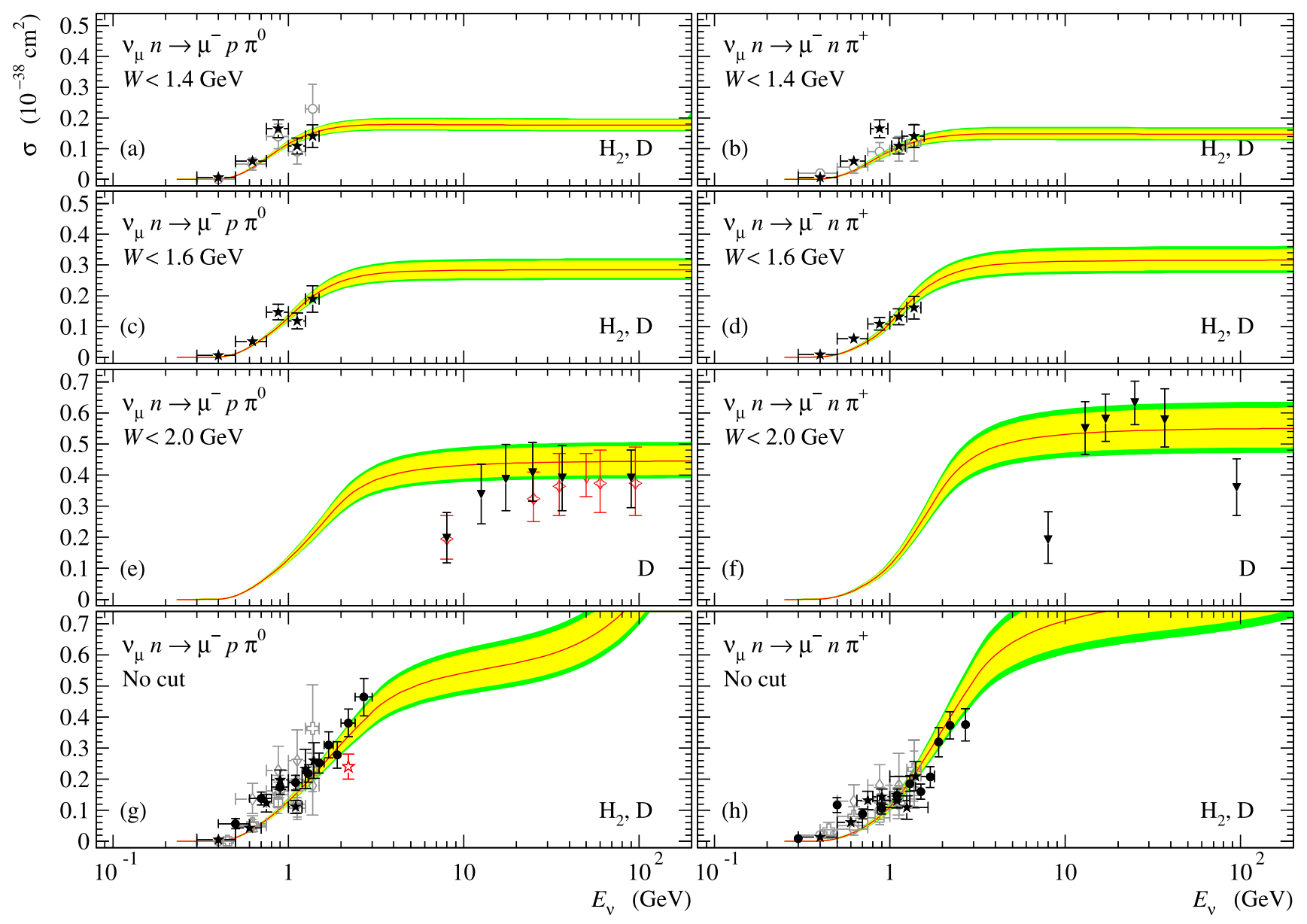

$\diamond$ Derrick et al., ANL 1974

↔ Barish et al., ANL 1976

$\square$ Barnes et al., ANL 1978

- Barish et al., ANL 1979 $\star$ Radecky et al., ANL 1982

- Kitagaki et al., BNL 1986

v Barlag et al., CERN BEBC 1984 th Allasia et al., CERN BEBC 1990

is Krenz et al., CERN GGM $1978\left(\mathrm{C}_{3} \mathrm{H}_{8}-\mathrm{CF}_{3} \mathrm{Br}\right)$, $W<2.55 \mathrm{GeV}$, corrected to free nucleon

FIG. 6. Total cross sections of the reaction $\nu_{\mu} n \rightarrow \mu^{-} p \pi^{0}$ (a), (c), (e), (g), $\nu_{\mu} n \rightarrow \mu^{-} n \pi^{+}$(b), (d), (f), (h), as functions of neutrino energy predicted with $M_{A}$ and $f_{\mathrm{NRB}}$ obtained from the global fits for $\mathrm{H}_{2}$ and $\mathrm{D}$ data in comparison with the experimental data measured at ANL 1974 [45], 1976 [47], 1978 [49], 1982 [53], 1979 [50] BNL 1986 [58], and CERN GGM 1978 [68], BEBC 1984 [76], 1990 [80,85]. Original experimental data measured at ANL 1982 [53] for $W<1.4 \mathrm{GeV}$ and with no cut for $W$; BNL 1986 [58] with no cut for $W$, recalculated by Rodrigues et al. [84]. Titles of the reactions, targets, and experimental cuts on $W$ are given in the legends. Shaded bands around the curves show the joint uncertainty of the fitted value of $M_{A}$ and $f_{\mathrm{NRB}}$ correspond to $1 \sigma$ and $2 \sigma$ errors. 


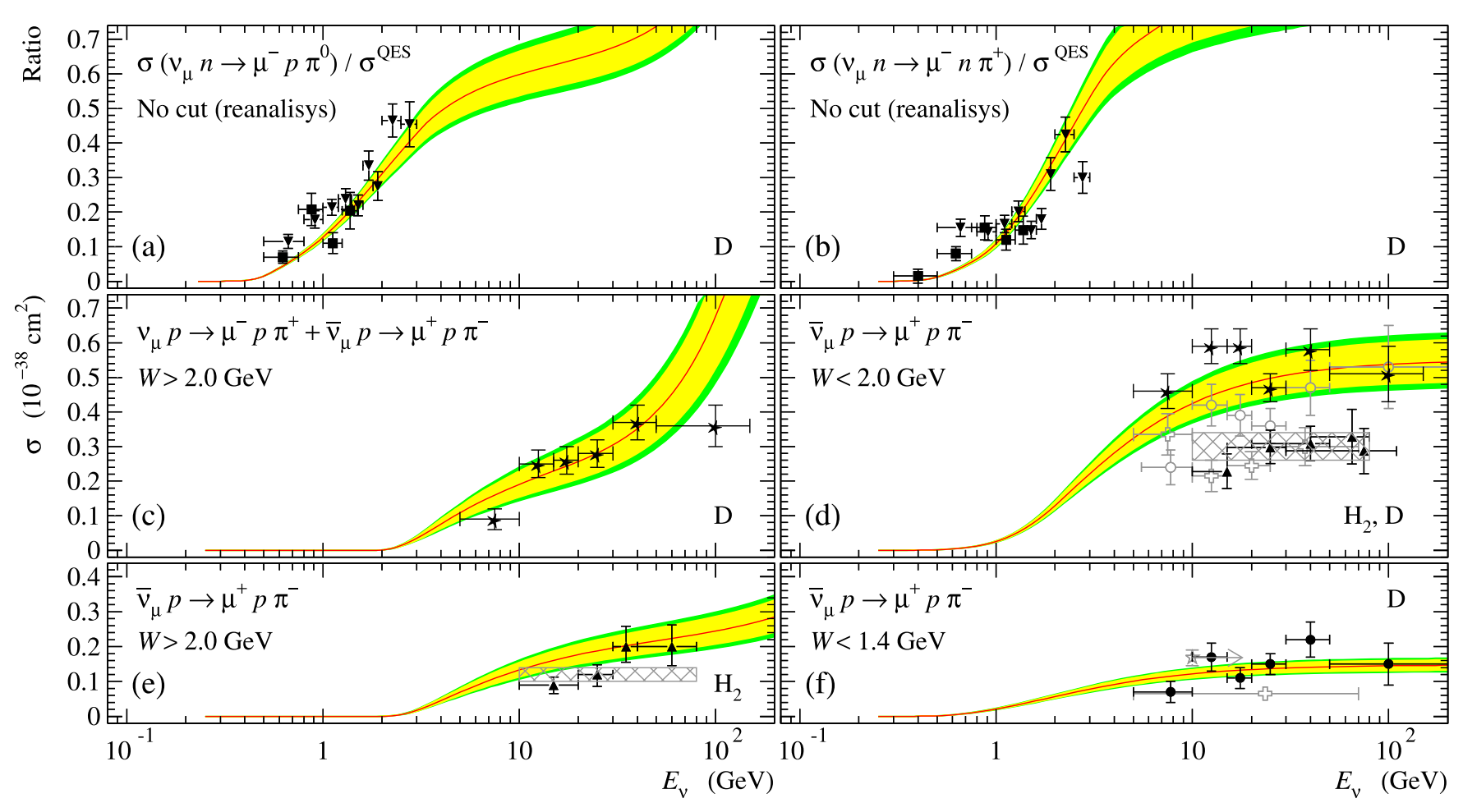

- Furuno et al., ANL 2003

- Furuno et al., BNL 2003

- $\circ$ Allasia et al., CERN BEBC 1983

is Jones et al., CERN BEBC 1989

Barish et al., FNAL 1980

* Allasia et al., CERN BEBC 1990

FIG. 7. Ratios of the total cross sections of the reactions $\nu_{\mu} n \rightarrow \mu^{-} p \pi^{0}$ (a) and $\nu_{\mu} n \rightarrow \mu^{-} n \pi^{+}$(b) to the quasielastic reaction $\nu_{\mu} n \rightarrow \mu^{-} p$, sum of the reactions $\nu_{\mu} p \rightarrow \mu^{-} p \pi^{+}$and $\bar{\nu}_{\mu} p \rightarrow \mu^{+} p \pi^{-}$(c), and total cross sections of the reaction $\bar{\nu}_{\mu} p \rightarrow \mu^{+} p \pi^{-}(\mathrm{d})-(\mathrm{f})$ predicted with $M_{A}$ and $f_{\mathrm{NRB}}$ obtained from the global fits for $\mathrm{H}_{2}$ and $\mathrm{D}$ data in comparison with the experimental data measured at ANL and BNL [81-83], FNAL 1980 [62] (measured with cuts of $W<1.9 \mathrm{GeV}$ and $W<1.32 \mathrm{GeV}$ ), CERN BEBC 1990 [80], 1983 [75], 1986 [77], and 1989 [78]. Titles of the reactions, targets, and experimental cuts on $W$ are given in the legends. Notation for the solid curves and shaded bands is the same as in Fig. 6. 


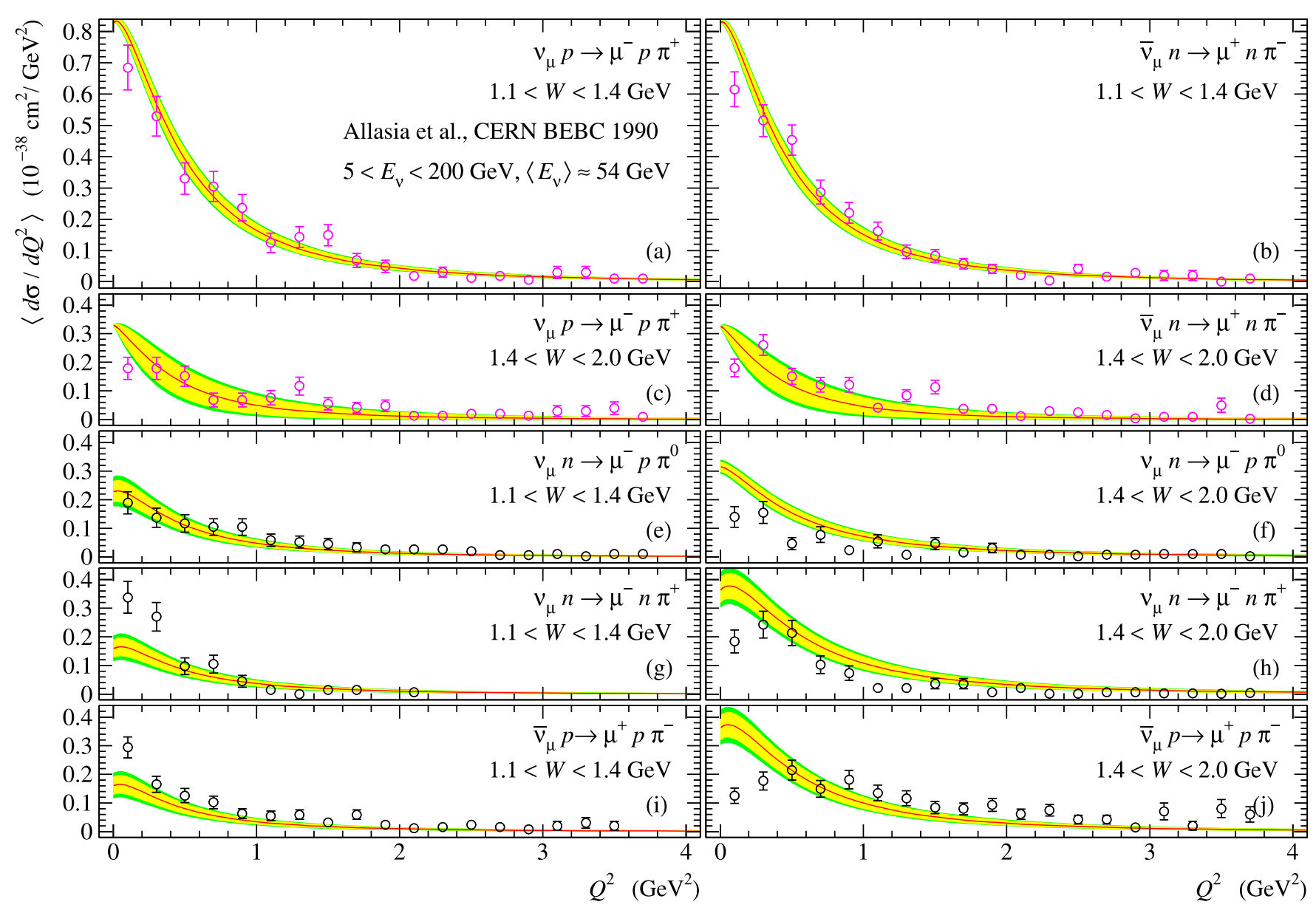

FIG. 8. Flux-weighted differential $Q^{2}$-dependent cross sections of the reactions $\nu_{\mu} p \rightarrow \mu^{-} p \pi^{+}$(a), (c), $\nu_{\mu} n \rightarrow \mu^{-} p \pi^{0}$ (e), (f), $\nu_{\mu} n \rightarrow$ $\mu^{-} n \pi^{+}(\mathrm{g}),(\mathrm{h}), \bar{\nu}_{\mu} n \rightarrow \mu^{+} n \pi^{-}(\mathrm{b}),(\mathrm{d})$, and $\bar{\nu}_{\mu} p \rightarrow \mu^{+} p \pi^{-}$(i), (j), predicted with $M_{A}$ and $f_{\mathrm{NRB}}$ obtained from the global fits for $\mathrm{H}_{2}$ and $\mathrm{D}$ data in comparison with the experimental data measured at CERN BEBC 1990 [80]. Neutrino and antineutrino fluxes are borrowed from [79]. Titles of the reactions, targets, and experimental cuts on $W$ and neutrino energy are given in the legends. Inner and outer shaded bands around the curves in panels (a), (c), (b), (d), demonstrate the uncertainty of the fitted value of $M_{A}$ corresponding to $1 \sigma$ and $2 \sigma$ errors, respectively. In the other panels the shaded bands demonstrate the joint uncertainty of the fitted value of $M_{A}$ and $f_{\mathrm{NRB}}$.

\section{CONCLUSIONS}

First, we recommend clarifying the RS model and its extensions by getting rid of the normalizations of the BreitWigner distributions to avoid unphysical quantities in calculations.

Next, the global fit of the experimental data on the total and differential CC cross sections for the $\nu_{\mu}$ and $\bar{\nu}_{\mu}$ reactions of the $1 \pi$-production through the nucleon and baryon resonances, measured in experiments on hydrogen and deuterium targets, allows one to determine the phenomenological parameters of the resonance current axialvector mass and the adjustable constant for fine-tuned noninterfering nonresonance background for the phenomenological models based on the RS approach. The axial mass extracted from the fit for only $\nu_{\mu}$ data yields the same result. The low number of the $\bar{\nu}_{\mu}$ data does not allow one to obtain a reliable result from the global fits; however the result does not contradict the global analysis. The cross sections predicted within the clarified KLN-BS model are in good agreement with the experimental data in the whole range of neutrino energy. The updated value of the axial mass is consistent with the previous one and describes well the data obtained in experiments with nuclear targets [20]. Modern experiments with deuterium and hydrogen targets operating with the $\nu_{\mu}$ and $\bar{\nu}_{\mu}$ beams are needed for a more precise determination of the parameters of the model.

Finally, we emphasize that the obtained resonance axial mass and adjustable nonresonance background are appropriate only for the RS model and its extensions in which the traditional normalization of the Breit-Wigner factors is abandoned. The alternative models for describing the resonance $1 \pi$-production reactions may require a different value of the axial mass and tune for the background induced by nonresonance reactions. 


\section{ACKNOWLEDGMENTS}

We are very grateful to Vadim A. Naumov for many useful discussions and for drawing our attention to the problem with calculating of the resonance Breit-Wigner normalization factors in the RS approach. We are very grateful to Satoshi Nakamura alerted us to that ANL and BNL data need to be corrected for nuclear effects and FSI. We thank Galina G. Sandukovskaya for proofreading of the text. Research of Igor D. Kakorin has been supported by the Russian Science Foundation Grant No. 18-12-00271.
[1] E. Fernandez-Martinez and D. Meloni, Importance of nuclear effects in the measurement of neutrino oscillation parameters, Phys. Lett. B 697, 477 (2011).

[2] D. Meloni and M. Martini, Revisiting the T2K data using different models for the neutrino-nucleus cross sections, Phys. Lett. B 716, 186 (2012).

[3] O. Benhar and N. Rocco, Nuclear effects in neutrino interactions and their impact on the determination of oscillation parameters, Adv. High Energy Phys. 2013, 1912702 (2013).

[4] P. Coloma and P. Huber, Impact of Nuclear Effects on the Extraction of Neutrino Oscillation Parameters, Phys. Rev. Lett. 111, 221802 (2013).

[5] P. Coloma, P. Huber, C.-M. Jen, and C. Mariani, Neutrinonucleus interaction models and their impact on oscillation analyses, Phys. Rev. D 89, 073015 (2014).

[6] C.-M. Jen, A. M. Ankowski, O. Benhar, A. P. Furmanski, L. N. Kalousis, and C. Mariani, Numerical implementation of lepton-nucleus interactions and its effect on neutrino oscillation analysis, Phys. Rev. D 90, 093004 (2014).

[7] M. Ericson and M. Martini, Neutrino versus antineutrino cross sections and CP violation, Phys. Rev. C 91, 035501 (2015).

[8] A. M. Ankowski, O. Benhar, C. Mariani, and E. Vagnoni, Effect of the $2 p 2 h$ cross-section uncertainties on an analysis of neutrino oscillations, Phys. Rev. D 93, 113004 (2016).

[9] A. M. Ankowski and C. Mariani, Systematic uncertainties in long-baseline neutrino-oscillation experiments, J. Phys. G 44, 054001 (2017).

[10] U. Mosel, Neutrino event generators: foundation, status and future, J. Phys. G 46, 113001 (2019).

[11] K. S. Kuzmin, V. V. Lyubushkin, and V. A. Naumov, How to sum contributions into the total charged-current neutrinonucleon cross section, arXiv:hep-ph/0511308.

[12] K. S. Kuzmin, V. V. Lyubushkin, and V. A. Naumov, Finetuning parameters to describe the total charged-current neutrino-nucleon cross section, Phys. At. Nucl. 69, 1857 (2006).

[13] F. Ravndal, Weak production of nuclear resonances in a relativistic quark model, Nuovo Cimento A 18, 385 (1973).

[14] D. Rein and L. M. Sehgal, Neutrino-excitation of baryon resonances and single pion production, Ann. Phys. (N.Y.) 133, 79 (1981).

[15] D. Rein, Angular distribution in neutrino induced single pion production processes, Z. Phys. C 35, 43 (1987).

[16] R. P. Feynman, M. Kislinger, and F. Ravndal, Current matrix elements from a relativistic quark model, Phys. Rev. D 3, 2706 (1971).
[17] M. Derrick et al., Simple charged-current channels in $\nu-\mathrm{D}_{2}$ interactions, in Proceedings of the Topical Conference on Neutrino Physics at Accelerators, Oxford, England, UK, July 4-7, 1978, edited by A. G. Michette and P. B. Renton (Science Research Council, Rutherford Laboratory, Chilton, England, UK, 1978), pp. 58-66.

[18] M. Rollier (Gargamelle Antineutrino Collaboration), Recent results from the Gargamelle anti-neutrino propane experiment at the CERN PS, in Proceedings of the Neutrino physics at accelerators, Topical Conference on Neutrino Physics, Oxford, England, UK, July 4-7, 1978, edited by A. G. Michette and P. B. Renton (Science Research Council, Rutherford Laboratory, Chilton, England, UK, 1978), pp. 68-74.

[19] M. Dewit, Experimental study of the reaction $\nu n \rightarrow \mu^{-} p$, in Proceedings of the Neutrino physics at accelerators, Topical Conference on Neutrino Physics, Oxford, England, UK, July 4-7, 1978, edited by A. G. Michetteand P. B. Renton (Science Research Council, Rutherford Laboratory, Chilton, England, UK, 1978), pp. 75-77.

[20] K. S. Kuzmin, V. V. Lyubushkin, and V. A. Naumov, Axial masses in quasielastic neutrino scattering and single-pion neutrinoproduction on nucleons and nuclei, Acta Phys. Pol. B 37, 2337 (2006), https://inspirehep.net/literature/719586.

[21] C. Andreopoulos et al. (GENIE Collaboration), The GENIE neutrino Monte Carlo generator, Nucl. Instrum. Methods Phys. Res., Sect. A 614, 87 (2010).

[22] C. Andreopoulos et al., The GENIE neutrino Monte Carlo generator: Physics and user manual, version 2.10.0, arXiv: 1510.05494.

[23] C. L. McGivern et al. (MINER $\nu$ A Collaboration), Cross sections for $\nu_{\mu}$ and $\bar{\nu}_{\mu}$ induced pion production on hydrocarbon in the few $\mathrm{GeV}$ region using MINER $\nu \mathrm{A}$, Phys. Rev. D 94, 052005 (2016).

[24] P. Adamson et al. (MINOS Collaboration), Study of quasielastic scattering using charged-current $\nu_{\mu}$-iron interactions in the MINOS near detector, Phys. Rev. D 91, 012005 (2015).

[25] K. Abe et al. (T2K Collaboration), The T2K experiment, Nucl. Instrum. Methods Phys. Res., Sect. A 659, 106 (2011).

[26] K. S. Kuzmin, V. V. Lyubushkin, and V. A. Naumov, Lepton polarization in neutrino nucleon interactions, Mod. Phys. Lett. A 19, 2815 (2004).

[27] K. S. Kuzmin, V. V. Lyubushkin, and V. A. Naumov, Extended Rein-Sehgal model for tau lepton production, Nucl. Phys. B, Proc. Suppl. 139, 158 (2005).

[28] C. Berger and L. M. Sehgal, Lepton mass effects in single pion production by neutrinos, Phys. Rev. D 76, 113004 (2007). 
[29] K. M. Graczyk and J. T. Sobczyk, Lepton mass effects in weak charged current single pion production, Phys. Rev. D 77, 053003 (2008).

[30] K. M. Graczyk and J. T. Sobczyk, Form factors in the quark resonance model, Phys. Rev. D 77, 053001 (2008); Erratum, 79, 053001 (2008).

[31] M. Kabirnezhad, Improvement of single pion production for T2K experiment simulation tools, Ph.D. thesis, National Center for Nuclear Research, Warsaw, 2017.

[32] E. Hernandez, J. Nieves, and M. Valverde, Weak pion production off the nucleon, Phys. Rev. D 76, 033005 (2007).

[33] M. Kabirnezhad, Single pion production in neutrinonucleon interactions, Phys. Rev. D 97, 013002 (2018).

[34] M. Kabirnezhad, Single pion production in neutrinonucleon interaction, J. Phys. Soc. Jpn. Conf. Proc. 12, 010043 (2016).

[35] M. Kabirnezhad, Single pion production in neutrino reactions, J. Phys. Conf. Ser. 888, 012122 (2017).

[36] P. A. Zyla et al. (Particle Data Group), Review of particle physics, Prog. Theor. Exp. Phys. 2020, 083C01 (2020).

[37] C. Patrignani et al. (Particle Data Group), Review of particle physics, Chin. Phys. C 40, 100001 (2016).

[38] The normalizations of the resonance Breit-Wigner distributions according to the previous version of KLN-BS model are 0.957 for $P_{33}(1234), 0.784$ for $P_{11}(1450), 1.055$ for $S_{31}(1620), 0.935$ for $P_{33}(1640), 0.751$ for $D_{33}(1730), 1.229$ for $P_{31}(1920), 0.635$ for $F_{35}(1920), 0.710$ for $F_{37}(1950)$, 1.285 for $P_{33}(1960), 1.008$ for $D_{13}(1525), 1.067$ for $S_{11}(1540), 1.051$ for $S_{11}(1640), 1.165$ for $D_{13}(1670)$, 1.024 for $D_{15}(1680), 0.912$ for $F_{15}(1680), 1.349$ for $P_{11}(1710), 1.301$ for $P_{13}(1740)$, and 0.619 for $F_{17}(1970)$.

[39] L. A. Ahrens et al., Determination of the neutrino fluxes in the Brookhaven wide-band beams, Phys. Rev. D 34, 75 (1986).

[40] F. James, MINUIT - function minimization and error analysis: Reference manual version 94.1, Report No. CERN-D506, 1994.

[41] F. James and M. Roos, MINUIT - a system for function minimization and analysis of the parameter errors and correlations, Comput. Phys. Commun. 10, 343 (1975).

[42] A. V. Baranov, N. A. Balashov, N. A. Kutovskiy, and R. N. Semenov, JINR cloud infrastructure evolution, Phys. Part. Nucl. Lett. 13, 672 (2016).N. A. Balashov et al., JINR cloud service for scientific and engineering computations, Mod. Inf. Tech. and IT-Ed. 14, 61 (2018).

[43] J. Campbell et al., Study of the Reaction $\nu p \rightarrow \mu^{-} p \pi^{+}$, Phys. Rev. Lett. 30, 335 (1973).

[44] P. A. Schreiner and F. Von Hippel, $\nu p \rightarrow \mu^{-} \Delta^{++}$: Comparison with Theory, Phys. Rev. Lett. 30, 339 (1973).

[45] M. Derrick, Charged current neutrino reactions in the resonance region, in Proceedings of the 17th International Conference on High-Energy Physics (ICHEP 1974), London, England, UK, July 1-10, 1974, edited by J. R. Smith (Science Research Council, Rutherford Laboratory, Chilton, England, UK, 1975), pp. II.166-170.

[46] D. H. Perkins, Review of neutrino experiments, in Proceedings of the International Symposium on Lepton and Photon Interactions at High Energies, Leland Stanford Junior University, Stanford, California, USA, August 21-27,
1975, edited by T. W. Kirk (SLAC National Accelerator Laboratory, Stanford, California, USA, 1975), pp. 571-603.

[47] S. J. Barish et al., Study of the Isospin Properties of SinglePion Production by Neutrinos, Phys. Rev. Lett. 36, 179 (1976).

[48] S. J. Barish et al., Study of neutrino interactions in hydrogen and deuterium: Description of the experiment and study of the reaction $\nu+d \rightarrow \mu^{-}+p+p_{s}$, Phys. Rev. D 16, 3103 (1977).

[49] V. E. Barnes et al., Study of the isospin properties of singlepion production by neutrinos, in Proceedings of the 6th International Conference on Neutrino Physics (Neutrino 1978), Purdue University, West Lafayette, Indiana, USA, Apr 28-May 2, 1978, edited by E. C. Fowler (Purdue University Press, West Lafayette, Indiana, USA, 1978), pp. C56-C63.

[50] S. J. Barish et al., Study of neutrino interactions in hydrogen and deuterium. II. Inelastic charged-current reactions, Phys. Rev. D 19, 2521 (1979).

[51] M. Derrick et al., Study of the reaction $\nu n \rightarrow \nu p \pi^{-}$, Phys. Lett. 92B, 363 (1980); Erratum, Phys. Lett. 95B, 461 (1980).

[52] M. Derrick et al., Study of single pion production by weak neutral currents in low-energy $\nu d$ interactions, Phys. Rev. D 23, 569 (1981).

[53] G. M. Radecky et al., Study of single-pion production by weak charged currents in low-energy $\nu d$ interactions, Phys. Rev. D 25, 1161 (1982); Erratum, Phys. Rev. D 26, 3297 (1982).

[54] W.-Y. Lee et al., Single Pion Production in Neutrino and Anti-Neutrino Reactions, Phys. Rev. Lett. 38, 202 (1977).

[55] G. K. Fanourakis, L. K. Resvanis, G. Grammatikakis, P. Tsilimigras, A. Vayaki, U. Camerini, W. F. Fry, R. J. Loveless, J. H. Mapp, and D. D. Reeder, Study of lowenergy anti-neutrino interactions on protons, Phys. Rev. D 21, 562 (1980).

[56] N. J. Baker, A. M. Cnops, P. L. Connolly, S. A. Kahn, M. J. Murtagh, R. B. Palmer, N. P. Samios, and M. Tanaka, Study of the isospin structure of single pion production in charged current neutrino interactions, Phys. Rev. D 23, 2495 (1981).

[57] N. J. Baker et al., Exclusive neutral-current reaction $\nu_{\mu} n \rightarrow \nu_{\mu} p \pi^{-}$in the BNL 7-foot deuterium bubble chamber, Phys. Rev. D 28, 2900 (1983).

[58] T. Kitagaki et al., Charged current exclusive pion production in neutrino deuterium interactions, Phys. Rev. D 34, 2554 (1986).

[59] R. T. Ross (Fermilab-Hawaii-Berkeley-Michigan Collaboration), A study of the reaction $\nu p \rightarrow \mu^{-} p \pi^{+}$, in Proceedings of the 6th International Conference on Neutrino Physics (Neutrino 1978), Purdue University, West Lafayette, Indiana, USA, Apr 28-May 2, 1978, edited by E. C. Fowler (Purdue University Press, West Lafayette, Indiana, USA, 1978), pp. 929-938.

[60] J. Bell et al., Study of the Reaction $\nu p \rightarrow \mu^{-} \Delta^{++}$at High Energies and Comparisons with Theory, Phys. Rev. Lett. 41, 1012 (1978).

[61] J. Bell et al., Cross-Section Measurements for the Reactions $\nu p \rightarrow \mu^{-} p \pi^{+}$and $\nu p \rightarrow \mu^{-} p K^{+}$at High Energies, Phys. Rev. Lett. 41, 1008 (1978). 
[62] S. J. Barish et al., Study of the reaction $\bar{\nu} p \rightarrow \mu^{+} p \pi^{+}$, Phys. Lett. 91B, 161 (1980).

[63] C. Franzinetti, Neutrino interactions in the CERN heavyliquid bubble-chamber, Technical Report CERN Yellow Report No. 66-13, 1966.

[64] E. C. M. Young, High-energy neutrino interactions, Technical Report CERN Yellow Report No. 67-12, 1967.

[65] I. Budagov et al., Single pion production by neutrinos on free protons, Phys. Lett. 29B, 524 (1969).

[66] F. J. Hasert et al., Neutral pion production by weak neutral currents in neutrino and antineutrino reactions, Phys. Lett. B 59B, 485 (1975).

[67] P. Schmid (Aachen-Bonn-CERN-Munich-Oxford Collaboration), First results from a $\nu p$ experiment at CERN, Conf. Proc. C 780428, 939 (1978), https://inspirehep.net/ literature/137405.

[68] W. Krenz et al. (Gargamelle Neutrino Propane, AachenBrussels-CERN-Ecole Poly-Orsay-Padua Collaborations), Experimental study of exclusive one-pion production in all neutrino-induced neutral current channels, Nucl. Phys. B135, 45 (1978).

[69] W. Lerche et al., Experimental study of the reaction $\nu_{\mu} p \rightarrow \mu^{-} p \pi^{+}$(Gargamelle Neutrino Propane experiment), Phys. Lett. 78B, 510 (1978).

[70] M. Pohl (Aachen-Brussels-CERN-Ecole PolytechniqueOrsay-Padua Collaboration), Experimental study of one pion production by the weak charged current, in Proceedings of the Topical Conference on Neutrino Physics at Accelerators, Oxford, England, UK, July 4-7, 1978, edited by A. G. Michette and P. B. Renton (Science Research Council, Rutherford Laboratory, Chilton, England, UK, 1978), pp. 78-82.

[71] M. Pohl et al., Experimental study of single pion production in charged current neutrino interactions, Lett. Nuovo Cimento 24, 540 (1979).

[72] T. Bolognese, Study of antineutrino interactions with charged current pion production, Ph.D. thesis, Strasbourg, CRN, 1978.

[73] T. Bolognese, J. P. Engel, J. L. Guyonnet, and J. L. Riester, Single pion production in antineutrino induced charged current interactions, Phys. Lett. 81B, 393 (1979).

[74] P. Allen et al. (Aachen-Bonn-CERN-München-Oxford Collaboration), Single $\pi^{+}$production in charged current neutrino-hydrogen interactions, Nucl. Phys. B176, 269 (1980).

[75] D. Allasia et al. (Amsterdam-Bergen-Bologna-PadovaPisa-Saclay-Torino Collaboration), Single pion production in charged current $\bar{\nu} D$ interactions at high energies, Z. Phys. C 20, 95 (1983).

[76] S. J. M. Barlag, Quasielastic interactions and one pion production by neutrinos and antineutrinos on a deuterium target, Ph.D. thesis, Amsterdam University, 1984.

[77] P. Allen et al. (Aachen-Birmingham-Bonn-CERN-LondonMunich-Oxford Collaboration), A study of single-meson production in neutrino and antineutrino charged-current interactions on protons, Nucl. Phys. B264, 221 (1986).

[78] G. T. Jones et al. (Birmingham-CERN-Imperial CollegeMünchen (MPI)-Oxford University College (WA21 Collaboration)), Experimental test of the PCAC hypothesis in the reactions $\nu_{\mu} p \rightarrow \mu^{-} p \pi^{+}$and $\bar{\nu}_{\mu} p \rightarrow \mu^{+} p \pi^{-}$in the $\Delta$ (1232) region, Z. Phys. C 43, 527 (1989).

[79] D. Allasia et al. (Amsterdam-Bergen-Bologna-PaduaPisa-Saclay-Torino Collaboration), Measurement of the $\nu_{\mu}$ and $\bar{\nu}_{\mu}$-nucleon charged current total cross-sections, and the ratio of $\nu_{\mu}$ neutron to $\nu_{\mu}$ proton charged current total cross-section, Nucl. Phys. B239, 301 (1984).

[80] D. Allasia et al., Investigation of exclusive channels in $\nu / \bar{\nu}$-deuteron charged current interactions, Nucl. Phys. B343, 285 (1990).

[81] K. Furuno et al., BNL 7-foot bubble chamber experiment: Neutrino deuterium interactions, in Proceedings of the 2nd International Workshop on Neutrino-Nucleus Interactions in the Few GeV Region (NuInt 2002), Irvine, California, USA, December 12-15, 2002 (North Holland Publishing Co., Amsterdam, 2002).

[82] M. Sakuda and E. F. Paschos, Single pion production in neutrino-nucleus interactions in the few $\mathrm{GeV}$ region, in Proceedings of the 2nd International Workshop on Neutrino-Nucleus Interactions in the Few GeV Region (NuInt 2002), Irvine, California, USA, December 12-15, 2002 (North Holland Publishing Co., Amsterdam, 2002).

[83] M. Sakuda, Study of neutrino-nucleus interactions for neutrino oscillations, in Proceedings of the 4th Workshop on Neutrino Oscillations and their Origin (NOON 2003), Ishikawa Kousei Nenkin Kaikan, Kanazawa, Japan, February 10-14, 2003, edited by Y. Suzuki, M. Nakahata, Y. Itow, M. Shiozawa, and Y. Obayashi (World Scientific Publishing Co Pte Ltd, Singapore, 2004), pp. 253-260.

[84] P. Rodrigues, C. Wilkinson, and K. McFarland, Constraining the GENIE model of neutrino-induced single pion production using reanalyzed bubble chamber data, Eur. Phys. J. C 76, 474 (2016).

[85] E. A. Hawker, Single pion production in low energy neutrino-carbon, in Proceedings of the 2nd International Workshop on Neutrino-Nucleus Interactions in the Few GeV Region (NuInt 2002), Irvine, California, USA, December 12-15, 2002 (North Holland Publishing Co., Amsterdam, 2002).

[86] I. D. Kakorin, K. S. Kuzmin, and V. A. Naumov, A unified empirical model for quasielastic interactions of neutrino and antineutrino with nuclei, Phys. Part. Nucl. Lett. 17, 265 (2020).

[87] B. C. Barish et al., Measurements of $\nu_{\mu} N$ and $\bar{\nu}_{\mu} N$ Charged Current Total Cross Sections, Phys. Rev. Lett. 39, 1595 (1977).

[88] P. C. Bosetti et al. (Aachen-Bonn-CERN-London-OxfordSaclay), Total cross sections for charged current neutrino and antineutrino interactions in $\mathrm{BEBC}$ in the energy range 20-200 GeV, Phys. Lett. 70B, 273 (1977).

[89] M. Glück, E. Reya, and A. Vogt, Dynamical parton distributions revisited, Eur. Phys. J. C 5, 461 (1998).

[90] H. J. Grabosch et al. (SKAT Collaboration), Cross section measurements of single pion production in charged current neutrino and antineutrino interactions, Z. Phys. C 41, 527 (1989).

[91] S. X. Nakamura, H. Kamano, and T. Sato, Impact of final state interactions on neutrino-nucleon pion production cross sections extracted from neutrino-deuteron reaction data, Phys. Rev. D 99, 031301 (2019). 